\title{
Asymptotic Properties for Weighted Averages of Longitudinal Dependent Data
}

\author{
Brahima Soro $^{1,2}$, Ouagnina Hilii ${ }^{2} \&$ Youssouf Diagana ${ }^{1}$ \\ ${ }^{1}$ Laboratoire de Mathématiques, Informatique et Applications, Université Nangui Abrogoua, 02 BP 801 Abidjan 02, Côte \\ d'Ivoire \\ ${ }^{2}$ Laboratoire de Mathématiques et des Nouvelles Technologies de l'Information, Institut National Polytechnique Félix \\ Houphouët-Boigny de Yamoussoukro, Côte d'Ivoire
}

Correspondence: Ouagnina Hili, BP 1911 Yamoussoukro, Côte d'Ivoire. E-mail: o_hili@yahoo.fr

Received: July 9, 2018 Accepted: October 5, 2018 Online Published: October 15, 2018

doi:10.5539/ijsp.v7n6p100

URL: https://doi.org/10.5539/ijsp.v7n6p100

\begin{abstract}
This paper presents a set of normality general results for kernel weighted averages. We extend existing literature for independent data (Yao, 2007) to stationary dependent longitudinal data. The asymptotic properties of proposed weighted averages are investigate under $\alpha$-mixing conditions. These results are useful for covariance function estimation based on nonparametric kernel method.
\end{abstract}

Keywords: longitudinal data, strong mixing data, weighted averages, covariance estimation, stationary process

\section{Introduction}

We present a set of asymptotic normality results of real-valued function that we assume to be formed by weighted averages of longitudinal data. Since it's well known that the most commonly nonparametric kernel type estimators are written as kernel weighted averages, our results take in account a large class of estimators (Nadaraya, 1964; Watson, 1964; Stone, 1977; Müller, 1984).

Recently, Yao (Yao, 2007) has given general normality results for some function of kernel averages formed by longitudinal independent data. He has applied his general result to covariance function estimator to derive its asymptotic distribution. Soro \& Hili (Soro \& Hili, 2012) have generalized the results of Yao (Yao, 2007) to three-dimensional context. The data were equally independent.

In this paper, we extend the two-dimensional general result (Yao, 2007) to dependent longitudinal data. Our main results are the asymptotic normality of a sample averages of some function that we suppose to be formed by longitudinal data. We suppose that the data are strongly mixing. The results we provide are applicable to covariance function kernel estimator to derive its asymptotic distribution under alpha-mixing conditions.

In Section 2, we introduce the model as well as assumptions that are necessary in deriving the main results of this paper. Section 3 presents main results of the paper.

\section{Model and Some Assumptions}

Let $\left\{\left(X_{i r}, U_{i r}, T_{i r}\right), 1 \leq i \leq n, 1 \leq r \leq N\right\}$ be $n \times N$ random variables, identically distributed as the random triple $(X, U, T)$ with values in $\mathbb{R} \times \mathbb{R} \times \mathrm{T}$, where $\mathrm{T}$ is such that $\mathrm{T}=[0, \mathcal{T}]$ with $\mathcal{T}<\infty$.

For the multi-index of integers $\lambda=\left(\lambda_{1}, \lambda_{2}\right)$ and $k=\left(k_{1}, k_{2}\right)$, let define $|\lambda|=\lambda_{1}+\lambda_{2},|k|=k_{1}+k_{2} ; \lambda !=\lambda_{1} ! \lambda_{2}$ ! and $k !=k_{1} ! k_{2} !$

We consider a model for repeated measurements, which is typically used for longitudinal data treatment :

$$
U_{i r}=X_{i}\left(T_{i r}\right)+\epsilon_{i r}, \quad 1 \leq i \leq n, 1 \leq r \leq N .
$$

In the model (1), $U_{i r}$ is referred to the $r$-th observation of the random variable $X_{i}$, made at the random time $T_{i r}$. Assume that

- the number of observations $N(n)$ depends on the sample size $n$. For simplicity, $N(n)$ will be noted $N$.

- $X$ takes values in a probability space $(\Omega, \mathcal{A}, \mathbb{P})$ whereas $U$ is a real random variable.

- the observation times $T_{i r}$ are i.i.d. with a marginal density $f_{0}(t)$. 
For $r \neq s$, and $(r, s) \neq\left(r^{\prime}, s^{\prime}\right)$, we define the joint probability densities as follow.

Let $f_{1}\left(t_{1}, t_{2}\right)$ be the joint density of $\left(T_{r}, T_{s}\right), g(t, u)$ the density of $\left(T_{r}, U_{s}\right), g_{1}\left(t_{1}, t_{2}, u_{1}, u_{2}\right)$ be the joint density of $\left(T_{r}, T_{s}, U_{r}, U_{s}\right)$ and $g_{2}\left(t_{1}, t_{2}, t_{1}^{\prime}, t_{2}^{\prime}, u_{1}, u_{2}, u_{1}^{\prime}, u_{2}^{\prime}\right)$ be the joint density of $\left(T_{r}, T_{s}, T_{r^{\prime}}, T_{s^{\prime}}, U_{r}, U_{s}, U_{r^{\prime}}, U_{s^{\prime}}\right)$.

We consider averages of longitudinal data of the form:

$$
\begin{aligned}
\hat{\Theta}_{n, \ell} & =\hat{\Theta}_{n, \ell}\left(t_{1}, t_{2}\right) \\
& =\frac{1}{n N(N-1) h_{n, K}^{|\lambda|+3}} \sum_{i=1}^{n} \sum_{1 \leq r \neq s \leq N} \psi_{\ell}\left(T_{i r}, T_{i s}, U_{i r}, U_{i s}\right) K\left(\frac{t_{1}-T_{i r}}{h_{n, K}}, \frac{t_{2}-T_{i s}}{h_{n, K}}\right),
\end{aligned}
$$

for $1 \leq \ell \leq L$, where $h_{n, K}$ is a bandwidth, $K: \mathbb{R}^{2} \longrightarrow \mathbb{R}$ is a kernel function and $\psi_{\ell}: \mathbb{R}^{2} \longrightarrow \mathbb{R}$ are real functions. Let

$$
\begin{aligned}
\vartheta_{\ell}\left(t_{1}, t_{2}\right) & =\|K\|_{2}^{2} \int_{\mathbb{R}^{2}} \psi_{\ell}^{2}\left(t_{1}, t_{2}, u_{1}, u_{2}\right) g_{1}\left(t_{1}, t_{2}, u_{1}, u_{2}\right) d u_{1} d u_{2}, \\
\theta_{\ell}\left(t_{1}, t_{2}\right) & =\frac{d^{|\lambda|}}{d t_{1}^{\lambda_{1}} d t_{2}^{\lambda_{2}}} \int_{\mathbb{R}^{2}} \psi_{\ell}\left(t_{1}, t_{2}, u_{1}, u_{2}\right) g_{1}\left(t_{1}, t_{2}, u_{1}, u_{2}\right) d u_{1} d u_{2}, \\
\mathbf{B}\left(t_{1}, t_{2}\right) & =\frac{(-1)^{|k|}}{k !}\left\{\int_{\mathbb{R}^{2}} v^{k_{1}} w^{k_{2}} K(v, w) d v d w \frac{d^{|k|}}{d t_{1}^{k_{1}} d t_{2}^{k_{2}}} \int_{\mathbb{R}^{2}} \psi_{\ell}\left(t_{1}, t_{2}, u_{1}, u_{2}\right) g_{1}\left(t_{1}, t_{2}, u_{1}, u_{2}\right) d u_{1} d u_{2}\right\}, \text { for } 1 \leq \ell \leq L .
\end{aligned}
$$

Let $\mathcal{N}_{\left(t_{1}, t_{2}\right)}$ be a neighborhood of $\left(t_{1}, t_{2}\right) \in[0 ; \mathcal{T}]^{2}$.

Now, we introduce the following basic assumptions that are necessary in deriving the main result of this paper.

(H1) (i) The kernel $K$ is symmetric with a compact support.

(ii) $\|K\|_{2}^{2}=\int_{\mathbb{R}^{2}} K^{2}(v, w) d v d w<\infty$.

(iii) $K$ is a kernel function of order $(|\lambda|,|k|)$, that is,

$$
\int_{\mathbb{R}^{2}} v^{l_{1}} w^{l_{2}} K(v, w) d v d w=\left\{\begin{aligned}
0, & & 0 \leq|l|<|k|,|l| \neq|v| . \\
(-1)^{|v|} v !, & & |l|=|v|, \\
C, & & |l|=|k| .
\end{aligned}\right.
$$

where $C$ is a non null constant.

(H2) The bandwidth $h_{n, K}$ satisfies,

(i) $h_{n, K} \longrightarrow 0, n N(N-1) h_{n, K}^{|\lambda|+2} \longrightarrow \infty, n N(N-1) h_{n, K}^{2|\lambda|+2} \longrightarrow a^{2}$, where $a$ is a positive constant, as $n \longrightarrow+\infty$.

(ii) $h_{n, K}^{|\lambda|+2} \longrightarrow \infty, n h_{n, K}^{|\lambda|+2} \longrightarrow \infty$ and $N(N-1) h_{n, K}^{|\lambda|} \longrightarrow \infty$, as $n \longrightarrow \infty$.

(H3) (i) $\frac{d^{k l}}{d u^{k} d v^{k_{2}} d w^{k_{3}}} f_{1}(v, w)$ exists and is continuous for $(v, w) \in \mathcal{N}_{\left(t_{1}, t_{2}\right)}$;

(ii) $g_{1}\left(v, w, u_{1}, u_{2}\right)$ is continuous for $(v, w) \in \mathcal{N}_{\left(t_{1}, t_{2}\right)}$, uniformly for $\left(u_{1}, u_{2}\right) \in \mathbb{R}^{2}$;

(H4) (i) $\frac{d^{k^{k l}}}{d v^{k_{1}} d w^{k_{2}}} g_{1}\left(v, w, u_{1}, u_{2}\right)$ exists and is continuous for $(v, w) \in \mathcal{N}_{\left(t_{1}, t_{2}\right)}$, uniformly for $\left(u_{1}, u_{2}\right) \in \mathbb{R}^{2}$;

(ii) $g_{2}\left(v, w, v^{\prime}, w^{\prime}, u_{1}, u_{2}, u_{1}^{\prime}, u_{2}^{\prime}\right)$ is continuous for $\left(v, w, v^{\prime}, w^{\prime}\right) \in \mathcal{N}_{\left(t_{1}, t_{2}\right)}^{2}$, uniformly for $\left(u_{1}, u_{2}\right) \in \mathbb{R}^{2}$.

(H5) The collection $\left\{\psi_{\ell}\right\}_{\ell=1, \ldots, l}$ of real functions $\psi_{\ell}: \mathbb{R}^{4} \rightarrow \mathbb{R}$ satisfies:

(i) $\psi_{\ell}\left(t_{1}, t_{2}, u_{1}, u_{2}\right)$ is continuous for $\left(t_{1}, t_{2}\right)$ uniformly for $\left(u_{1}, u_{2}\right) \in \mathbb{R}^{2}$,

(ii) $\frac{d^{k \mid}}{d t_{1}^{k_{1}} d t_{2}^{k_{2}}} \psi_{\ell}\left(t_{1}, t_{2}, u_{1}, u_{2}\right)$ exists for all arguments $\left(t_{1}, t_{2}, u_{1}, u_{2}\right) \in \mathbb{R}^{4}$.

Let $\mathcal{F}_{a}^{b}$ be the sigma algebra generated by the random variables $\left\{X_{i}, Y_{i}\right\}_{i=a}^{b}$.

The stationary process $\left\{X_{i}, Y_{i .}\right\}_{i=a}^{b}$ is called strongly mixing (Rosenblatt, 1956) if

$$
\alpha(j)=\sup _{t} \sup _{A \in \mathcal{F}_{-\infty}^{t}, B \in \mathcal{F}_{t+j}^{\infty}}|\mathbb{P}(A \cap B)-\mathbb{P}(A) \mathbb{P}(B)| \longrightarrow 0 \text {, as } j \longrightarrow \infty .
$$

(H6) The process $\left\{X_{i}, Y_{i}\right\}$ is strongly mixing with coefficient

$$
\sum_{j=1}^{\infty} j^{a}[\alpha(j)]^{1-2 / \delta}<\infty \text { for some } a>1-2 / \delta, \text { and some } \delta>2 .
$$


Comments on the assumptions. Assumptions (H1) and (H2) are technical conditions for the proofs. Assumptions (H3) and (H4) are regularity conditions for joint probability densities. Assumption (H6) is mixing condition verified by the process.

\section{Main Results: Consistency and Asymptotic Normality of Kernel Averages}

Before establishing the main results of the paper, we first prove the consistency and the asymptotic normality of weighted averages (2).

\subsection{Consistency of Kernel Averages}

In this part of paper, we establish the consistency of (2). The result is given in the following theorem.

Theorem 1 If assumptions (H1)-(H5) are satisfied, we have

$$
\hat{\Theta}_{n, \ell}\left(t_{1}, t_{2}\right) \stackrel{\mathbb{P}}{\longrightarrow} \theta_{\ell}\left(t_{1}, t_{2}\right),
$$

where $\theta_{\ell}\left(t_{1}, t_{2}\right)$ is defined in (4) for $\ell=1, \ldots, L$.

Proof. To establish the consistency of (8) we have to consider the following decomposition

$$
\mathbb{E}\left\{\left(\hat{\Theta}_{n, \ell}\left(t_{1}, t_{2}\right)-\theta_{\ell}\left(t_{1}, t_{2}\right)\right)^{2}\right\}=\operatorname{Var}\left\{\hat{\Theta}_{n, \ell}\left(t_{1}, t_{2}\right)\right\}+\left\{\mathbb{E}\left[\hat{\Theta}_{n, \ell}\left(t_{1}, t_{2}\right)\right]-\theta_{\ell}\left(t_{1}, t_{2}\right)\right\}^{2} .
$$

We denote by $\stackrel{\mathbb{L}^{2}}{\longrightarrow}$ (respectively $\stackrel{\mathbb{P}}{\longrightarrow}$ ) the convergence in $\mathbb{L}^{2}$ (resp. in probability) and we also recall that when (9) goes to zero, we have

$$
\hat{\Theta}_{n, \ell}\left(t_{1}, t_{2}\right) \stackrel{\mathbb{L}^{2}}{\longrightarrow} \theta_{\ell}\left(t_{1}, t_{2}\right) \text { implies } \hat{\Theta}_{n, \ell}\left(t_{1}, t_{2}\right) \stackrel{\mathbb{P}}{\longrightarrow} \theta_{\ell}\left(t_{1}, t_{2}\right) .
$$

- Let prove that the second term in (9) goes to 0 when $n$ goes to $+\infty$. We have

$$
\begin{aligned}
\mathbb{E} \hat{\Theta}_{n, \ell}\left(t_{1}, t_{2}\right) & =\frac{1}{n N(N-1) h_{n, K}^{|v|+2}} \mathbb{E}\left\{\sum_{i=1}^{n} \sum_{1 \leq r \neq s \leq N} \psi_{\ell}\left(T_{i r}, T_{i s}, U_{i r}, U_{i s}\right) K\left(\frac{t_{1}-T_{i r}}{h_{n, K}}, \frac{t_{2}-T_{i s}}{h_{n, K}}\right)\right\} \\
& =\frac{1}{N(N-1) h_{n, K}^{|v|+2}} \mathbb{E}\left\{\sum_{1 \leq r \neq s \leq N} \psi_{\ell}\left(T_{i r}, T_{i s}, U_{i r}, U_{i s}\right) K\left(\frac{t_{1}-T_{i r}}{h_{n, K}}, \frac{t_{2}-T_{i s}}{h_{n, K}}\right)\right\} \\
& =\frac{1}{h_{n, K}^{|v|+2}} \mathbb{E}\left\{\psi_{\ell}\left(T_{11}, T_{12 s}, U_{11}, U_{12}\right) K\left(\frac{t_{1}-T_{11}}{h_{n, K}}, \frac{t_{2}-T_{12}}{h_{n, K}}\right)\right\} \\
& =\theta_{\ell}\left(t_{1}, t_{2}\right)+\frac{(-1)^{|k|}}{k !}\left\{\int_{\mathbb{R}^{2}} v^{k_{1}} w^{k_{2}} K(v, w) d v d w\right. \\
& \left.\times \frac{d^{|k|}}{d t_{1}^{k_{1}} d t_{2}^{k_{2}}} \int_{\mathbb{R}^{2}} \psi_{\ell}\left(t_{1}, t_{2}, u_{1}, u_{2}\right) g_{1}\left(t_{1}, t_{2}, u_{1}, u_{2}\right) d u_{1} d u_{2} \times h_{n, K}^{|k|-|\lambda|}\right\}+o\left(h_{n, K}^{|k|-|\lambda|}\right) \\
& =\theta_{\ell}\left(t_{1}, t_{2}\right)+\mathbf{B}\left(t_{1}, t_{2}\right) \times h_{n, K}^{|k|-|\lambda|}+o\left(h_{n, K}^{|k|-|\lambda|}\right) .
\end{aligned}
$$

Then,

$$
\mathbb{E} \hat{\Theta}_{n, \ell}\left(t_{1}, t_{2}\right)-\theta_{\ell}\left(t_{1}, t_{2}\right)=\mathbf{B}\left(t_{1}, t_{2}\right) \times h_{n, K}^{|k|-|\lambda|}+o\left(h_{n, K}^{|k|-|\lambda|}\right) .
$$

And it follows that

$$
\mathbb{E} \hat{\Theta}_{n, \ell}\left(t_{1}, t_{2}\right)-\theta_{\ell}\left(t_{1}, t_{2}\right) \rightarrow 0
$$

- Now, we prove that $\operatorname{Var}\left(\hat{\Theta}_{n, \ell}\left(t_{1}, t_{2}\right)\right) \longrightarrow 0$, as $n \longrightarrow \infty$.

Let

$$
\Gamma_{i, r, s}^{\ell}=\psi_{\ell}\left(T_{i r}, T_{i s}, U_{i r}, U_{i s}\right) K\left(\frac{t_{1}-T_{i r}}{h_{n, K}}, \frac{t_{2}-T_{i s}}{h_{n, K}}\right)
$$


Using the definition of the variance, we have

$$
\begin{aligned}
\operatorname{Var}\left(\hat{\Theta}_{n, \ell}\left(t_{1}, t_{2}\right)\right) & =\frac{1}{n N(N-1) h_{n, K}^{2|\lambda|+4}} \operatorname{Var}\left(\Gamma_{1,1,2}^{\ell}\right) \\
& +\frac{1}{\left\{n N(N-1) h_{n, K}^{|\lambda|+2}\right\}^{2}} \sum_{i=1}^{n} \sum_{\substack{i^{\prime}=1 \\
i \neq i^{\prime}}}^{n} \sum_{1 \leq r \neq s^{\prime} \leq N} \sum_{1 \leq r^{\prime} \neq s^{\prime} \leq N} \operatorname{Cov}\left(\Gamma_{i, r, s}^{\ell}, \Gamma_{i^{\prime}, r^{\prime}, s^{\prime}}^{\ell}\right) \\
& =\mathfrak{I}_{1}+\mathfrak{I}_{2} .
\end{aligned}
$$

$\rightsquigarrow$ Concerning $\mathfrak{I}_{1}$,

$$
\begin{aligned}
\mathfrak{I}_{1} & =\frac{1}{n N(N-1) h_{n, K}^{|\lambda|+2}} \\
& \times\left\{\mathbb{E}\left[\frac{1}{h_{n, K}^{|\lambda|+2}} \psi_{\ell}^{2}\left(T_{11}, T_{12}, U_{11}, U_{12},\right) K^{2}\left(\frac{t_{1}-T_{11}}{h_{n, K}}, \frac{t_{2}-T_{12}}{h_{n, K}}\right)\right]\right. \\
& \left.-\mathbb{E}^{2}\left[\frac{1}{h_{n, K}^{|\lambda|+2}} \psi_{\ell}^{2}\left(T_{11}, T_{12}, U_{11}, U_{12},\right) K^{2}\left(\frac{t_{1}-T_{11}}{h_{n, K}}, \frac{t_{2}-T_{12}}{h_{n, K}}\right)\right]\right\} \\
& =\frac{1}{n N(N-1) h_{n, K}^{|\lambda|+2}} \\
& \times \frac{1}{h_{n, K}^{|\lambda|+2}} \int_{\mathbb{R}^{4}} g_{1}\left(t, t^{\prime}, u_{1}, u_{2}\right) \psi_{\ell}^{2}\left(t, t^{\prime}, u_{1}, u_{2}\right) K^{2}\left(\frac{t_{1}-t}{h_{n, K}}, \frac{t_{2}-t^{\prime}}{h_{n, K}}\right) d t d t^{\prime} d u_{1} d u_{2} \\
& \left.-\left[\frac{1}{h_{n, K}^{|\lambda|+2}} \int_{\mathbb{R}^{4}} g_{1}\left(t, t^{\prime}, u_{1}, u_{2}\right) \psi_{\ell}\left(t, t^{\prime}, u_{1}, u_{2}\right) K\left(\frac{t_{1}-t}{h_{n, K}}, \frac{t_{2}-t^{\prime}}{h_{n, K}}\right) d t d t^{\prime} d u_{1} d u_{2}\right]^{2}\right\} .
\end{aligned}
$$

We have by changing variables

$$
\begin{aligned}
\mathfrak{I}_{1}= & \frac{1}{n N(N-1) h_{n, K}^{|\lambda|+2}}\left\{\frac{1}{h_{n, K}^{|\lambda|}} \int_{\mathbb{R}^{4}} g_{1}\left(t_{1}-h_{n, K} v, t_{2}-h_{n, K} w, u_{1}, u_{2}\right) \times\right. \\
& \psi_{\ell}^{2}\left(t_{1}-h_{n, K} v, t_{2}-h_{n, K} w, u_{1}, u_{2}\right) K^{2}(v, w) d v d w d u_{1} d u_{2} \\
- & h_{n, K}^{2}\left[\frac{1}{h_{n, K}^{|\lambda|}} \int_{\mathbb{R}^{4}} g_{1}\left(t_{1}-h_{n, K} v, t_{2}-h_{n, K} w, u_{1}, u_{2}\right)\right. \\
& \left.\left.\psi_{\ell}\left(t_{1}-h_{n, K} v, t_{2}-h_{n, K} w, u_{1}, u_{2}\right) K(v, w) d v d w d u_{1} d u_{2}\right]^{2}\right\} \\
= & \frac{1}{n N(N-1) h_{n, K}^{|\lambda|+2}}\left\{\vartheta_{\ell}\left(t_{1}, t_{2}\right)+o(1)\right\} \\
\longrightarrow & 0, n \longrightarrow+\infty .
\end{aligned}
$$

\section{$\rightsquigarrow$ Concerning $\mathfrak{I}_{2}$,}

put

$$
\Gamma_{i, r, s}^{\ell, 1,2}=\psi_{\ell}\left(T_{i r}^{(1)}, T_{i s}^{(2)}, U_{i r}^{(1)}, U_{i s}^{(2)}\right) K\left(\frac{t_{1}-T_{i r}^{(1)}}{h_{n, K}}, \frac{t_{2}-T_{i s}^{(2)}}{h_{n, K}}\right) .
$$

Given that triples $\left\{Y_{i j}, Y_{i k}, Y_{i l}\right\}$ and $\left\{Y_{i j^{\prime}}, Y_{i k^{\prime}}, Y_{i l^{\prime}}\right\}$ are independent and equidistributed then we can write

$$
\begin{aligned}
\mathfrak{I}_{2} & =\frac{\{N(N-1)\}^{2}}{\left\{n N(N-1) h_{n, K}^{|\lambda|+2}\right\}^{2}} \sum_{\substack{i=1 \\
i \neq i^{\prime}}}^{n} \sum_{i^{\prime}=1}^{n} \operatorname{Cov}\left(\Gamma_{i, 1,1}^{\ell, 1,2} ; \Gamma_{i^{\prime}, 2,2}^{\ell, 1,2}\right) \\
& =\frac{1}{n^{2} h_{n, K}^{2|\lambda|+4}} \sum_{\substack{i=1 \\
i \neq i^{\prime}}}^{n} \sum_{i^{\prime}=1}^{n} \operatorname{Cov}\left(\Gamma_{i, 1,1}^{\ell, 1,2} ; \Gamma_{i^{\prime}, 2,2}^{\ell, 1,2}\right) \\
& =\frac{1}{n^{2} h_{n, K}^{2|\lambda|+4}} \sum_{\substack{i=1 \\
i \neq i^{\prime}}}^{n} \sum_{i^{\prime}=1}^{n} \operatorname{Cov}\left(\Gamma_{i, \ell} ; \Gamma_{i^{\prime}, \ell}\right) .
\end{aligned}
$$


Let $\Lambda_{n}$ be a positive sequence tending to $\infty$ to be specified later on. Define

$$
S=\left\{\left(i, i^{\prime}\right): 0 \leq\left|i-i^{\prime}\right|<\Lambda_{n}, i, i^{\prime}=1, \ldots, n, i \neq i^{\prime}\right\} .
$$

Split (14) into two separate summations over index in $S$ and not in $S$. That is

$$
\mathfrak{I}_{2}=\mathfrak{I}_{21}+\mathfrak{I}_{22},
$$

where

$$
\begin{aligned}
& \mathfrak{I}_{21}=\frac{1}{n^{2} h_{n, K}^{2|\lambda|+4}}\left\{\sum_{i, i^{\prime}=1}^{n} \sum_{\left(i, i^{\prime}\right) \in S} \operatorname{Cov}\left(\Gamma_{i, \ell} ; \Gamma_{i^{\prime}, \ell}\right)\right\} \\
& \mathfrak{I}_{22}=\frac{1}{n^{2} h_{n, K}^{2|\lambda|+4}}\left\{\sum_{i, i^{\prime}=1}^{n} \sum_{\left(i, i^{\prime}\right) \notin S} \operatorname{Cov}\left(\Gamma_{i, \ell} ; \Gamma_{i^{\prime}, \ell}\right)\right\} .
\end{aligned}
$$

For (16), using Hölder's inequality, $\left|\operatorname{Cov}\left(\Gamma_{i, \ell}, \Gamma_{i^{\prime}, \ell}\right)\right| \leq\left(\mathbb{E} \Gamma_{i, \ell}^{2} \mathbb{E} \Gamma_{i^{\prime}, \ell}^{2}\right)^{1 / 2}+\left(\mathbb{E}\left|\Gamma_{i^{\prime}, \ell}\right|\right)^{2}$, it follows that

$$
\begin{aligned}
\left|\Im_{21}\right| & \leq \frac{1}{n^{2} h_{n, K}^{|\lambda|+2}} \sum_{i, i^{\prime}=1}^{n} \sum_{\left(i, i^{\prime}\right) \in S}\left\{\frac{1}{n^{2} h_{n, K}^{|\lambda|+2}}\left(\mathbb{E} \Gamma_{i, \ell}^{2} \mathbb{E} \Gamma_{i^{\prime}, \ell}^{2}\right)^{1 / 2}+\frac{1}{n^{2} h_{n, K}^{|\lambda|+2}}\left(\mathbb{E}\left|\Gamma_{i^{\prime}, \ell}\right|\right)^{2}\right\} \\
& =\frac{1}{n^{2} h_{n, K}^{|\lambda|+2}} \sum_{i, i^{\prime}=1}^{n} \sum_{\left(i, i^{\prime}\right) \in S}\left\{\vartheta_{\ell}\left(t_{1}, t_{2}\right)+o(1)\right\} .
\end{aligned}
$$

Since $\operatorname{Card}(S) \leq n \Lambda_{n}$, then

$$
\begin{aligned}
\left|\mathfrak{I}_{21}\right| & \leq \frac{n \Lambda_{n}}{n^{2} h_{n, K}^{|\lambda|+2}}\left\{\vartheta_{\ell}\left(t_{1}, t_{2}\right)+o(1)\right\} \\
& \leq \frac{\Lambda_{n}}{n h_{n, K}^{|\lambda|+2}}\left\{\vartheta_{\ell}\left(t_{1}, t_{2}\right)+o(1)\right\} .
\end{aligned}
$$

Clearly, if taking $\Lambda_{n}=(\ln \ln n)^{2} \ln n, h_{n, K}=\frac{\ln \ln n}{\ln n}$ in (18), one obtain

$$
\Lambda_{n} \longrightarrow \infty, h_{n, K}^{|\lambda|+2} \longrightarrow 0, n h_{n, K}^{|\lambda|+2} \longrightarrow \infty \text { and } \frac{\Lambda_{n}}{n h_{n, K}^{|\lambda|+2}} \longrightarrow 0,
$$

so

$$
\mathfrak{I}_{21} \longrightarrow 0, \text { as } n \longrightarrow+\infty \text {. }
$$

Turn to $\mathfrak{I}_{22}$. Applying Davydov's Lemma (see Hall \& Heyde, Corollary A.2), and assumption (H6) we have

Using (21)

$$
\begin{aligned}
\left|\operatorname{Cov}\left(\Gamma_{i, \ell}, \Gamma_{i^{\prime}, \ell^{\prime}}\right)\right| & \leq 8\left(\mathbb{E}\left|\Gamma_{i, \ell}\right|^{\delta}\right)^{2 / \delta}\left(\alpha\left(\left|i-i^{\prime}\right|\right)\right)^{1-2 / \delta} \\
& \leq 8 \operatorname{Const}\left[h_{n, K}^{|\lambda|+2}\right]^{2 / \delta}\left[\alpha\left(\left|i-i^{\prime}\right|\right)\right]^{1-2 / \delta} .
\end{aligned}
$$

$$
\begin{aligned}
\left|\Im_{22}\right| & \leq \frac{8 \text { Const. }\left[h_{n, K}^{|\lambda|+2}\right]^{2 / \delta}}{n^{2} h_{n, K}^{2|v|+4}} \sum_{i, i^{\prime}=1}^{n} \sum_{\left(i, i^{\prime}\right) \notin S}\left[\alpha\left(\left|i-i^{\prime}\right|\right)\right]^{1-2 / \delta} \\
& \leq \frac{8 \text { Const. }}{n^{2} h_{n, K}^{(|\lambda|+2)(2(1-1 / \delta))}} \sum_{i, i^{\prime}=1}^{n} \sum_{\left(i, i^{\prime}\right) \notin S}\left[\alpha\left(\left|i-i^{\prime}\right|\right)\right]^{1-2 / \delta} .
\end{aligned}
$$

Reducing the double sum above to a single sum, it follows that

$$
\begin{aligned}
\left|\mathfrak{I}_{22}\right| & \leq \frac{8 \text { Const. }}{n^{2} h_{n, K}^{(|\lambda|+2)(2(1-1 / \delta))}} \sum_{\ell=\Lambda_{n}+1}^{n} \ell^{a}[\alpha(\ell)]^{1-2 / \delta} \\
& \leq \frac{8 n \text { Const. }}{n^{2} h_{n, K}^{(|\lambda|+2)(2(1-1 / \delta))}} \sum_{\ell=\Lambda_{n}+1}^{n} \ell^{a}[\alpha(\ell)]^{1-2 / \delta} \\
& \leq \frac{8 \text { Const. }}{n h_{n, K}^{(|\lambda|+2)(2(1-1 / \delta))}} \sum_{\ell=\Lambda_{n}+1}^{\infty} \ell^{a}[\alpha(\ell)]^{1-2 / \delta} .
\end{aligned}
$$


Since $\delta>2$, it is easy to see that $(2-1 / \delta)>0$, by (H2) $n h_{n, K}^{|\lambda|+2} \longrightarrow \infty$ and applying (H6) in (22) it follows that

$$
\mathfrak{I}_{22} \quad \longrightarrow \quad 0 \text {, as } n \longrightarrow+\infty
$$

Finally $\operatorname{Var}\left(\hat{\Theta}_{n, \ell}\left(t_{1}, t_{2}\right)\right) \longrightarrow 0$, as $n \longrightarrow \infty$ via (13), (19) and (23).

This conclude the proof of theorem $1 \square$

\subsection{Asymptotic Normality of Kernel Averages}

Here, we give the asymptotic normality of (2) in the following theorem.

Theorem 2 If assumptions (H1)- (H5) and (H6) are satisfied, we have

$$
\sqrt{n N(N-1) h_{n, K}^{|\lambda|+2}}\left(\hat{\Theta}_{n, \ell}\left(t_{1}, t_{2}\right)-\mathbb{E} \hat{\Theta}_{n, \ell}\left(t_{1}, t_{2}\right)\right) \stackrel{\mathcal{D}}{\longrightarrow} \mathcal{N}\left(0, \vartheta_{\ell}\left(t_{1}, t_{2}\right)\right),
$$

where $\stackrel{\mathcal{D}}{\longrightarrow}$ denote the convergence in distribution.

Proof. We will establish the asymptotic normality of $\hat{\Theta}_{n, \ell}\left(t_{1}, t_{2}\right)-\mathbb{E}_{\Theta_{n, \ell}}\left(t_{1}, t_{2}\right)$ suitably normalized. We have

$$
\begin{gathered}
\sqrt{n N(N-1) h_{n, K}^{|\lambda|+2}}\left(\hat{\Theta}_{n, \ell}\left(t_{1}, t_{2}\right)-\mathbb{E} \hat{\Theta}_{n, \ell}\left(t_{1}, t_{2}\right)\right)=\frac{\sqrt{n N(N-1) h_{n, K}^{|\lambda|+2}}}{n N(N-1) h_{n, K}^{|\lambda|+2}} \sum_{i=1}^{n} \\
\sum_{1 \leq r \neq s \leq N}\left[\psi_{\ell}\left(T_{i r}, T_{i s}, U_{i r}, U_{i s}\right) K\left(\frac{t_{1}-T_{i r}}{h_{n, K}}, \frac{t_{2}-T_{i s}}{h_{n, K}}\right)\right. \\
\left.-\mathbb{E}_{\ell}\left(T_{i r}, T_{i s}, U_{i r}, U_{i s}\right) K\left(\frac{t_{1}-T_{i r}}{h_{n, K}}, \frac{t_{2}-T_{i s}}{h_{n, K}}\right)\right] \\
=\frac{1}{\sqrt{n N(N-1) h_{n, K}^{2}} \sum_{i=1}^{n} \sum_{1 \leq r \neq s \leq N}\left[\Gamma_{i, r, s}^{\ell}-\mathbb{E} \Gamma_{i, r, s}^{\ell}\right]} \\
=\sum_{i=1}^{n} \sum_{1 \leq r \neq s \leq N} \frac{1}{\sqrt{n N(N-1) h_{n, K}^{2}}}\left[\Gamma_{i, r, s}^{\ell}-\mathbb{E} \Gamma_{i, r, s}^{\ell}\right],
\end{gathered}
$$

where $\Gamma_{i, r, s}^{\ell}$ is defined in (12).

Denote

$$
\Xi_{i j k l}=\frac{1}{\sqrt{n N(N-1) h_{n, K}^{2}}} \Gamma_{i, r, s}^{\ell}
$$

Then

$$
\begin{aligned}
\sqrt{n N(N-1) h_{n, K}^{|\lambda|+2}}\left(\hat{\Theta}_{n, \ell}\left(t_{1}, t_{2}\right)-\mathbb{E} \hat{\Theta}_{n, \ell}\left(t_{1}, t_{2}\right)\right) & =\sum_{i=1}^{n} \sum_{1 \leq j \neq k \neq l \leq N}\left(\Xi_{i j k l}-\mathbb{E} \Xi_{i j k l}\right) \\
& =\sum_{i=1}^{n} \Xi_{n, i} .
\end{aligned}
$$

We now introduce Bernstein's big-block and small-block decomposition. We partition the set $\{1,2, \ldots, n\}$ into $2 k_{n}+1$ subsets with large blocks of size $u_{n}$ and small blocks of size $v_{n}$ and we set $k_{n}=\left\lfloor\frac{n}{u_{n}+v_{n}}\right\rfloor$, where $u_{n}=\left\lfloor n N(N-1) h_{n, K}^{|\lambda|+2}\right\rfloor$ and $v_{n}=o\left(n N(N-1) h_{n, K}^{|\lambda|+2}\right)$. The symbol L.」 is integer part. Using $(\mathrm{H} 2)$, one has

$$
\frac{v_{n}}{u_{n}} \longrightarrow 0, \frac{u_{n}}{n} \longrightarrow 0 \quad, \quad \frac{n N(N-1)}{u_{n} h_{n, K}^{2}} \longrightarrow 0, \frac{n}{u_{n}} \alpha\left(v_{n}\right) \longrightarrow 0, \text { as } n \longrightarrow+\infty
$$


Let $U_{m}, V_{m}$ and $W_{m}$ be defined as follows:

$$
\begin{aligned}
& U_{m}=\sum_{i=m\left(u_{n}+v_{n}\right)+1}^{m\left(u_{n}+v_{n}\right)+u_{n}} \Xi_{n, i}, \quad 0 \leq m \leq k_{n}-1 \\
& V_{m}=\sum_{i=m\left(u_{n}+v_{n}\right)+u_{n}+1}^{(m+1)\left(u_{n}+v_{n}\right)} \Xi_{n, i}, \quad 0 \leq m \leq k_{n}-1 \\
& W_{m}=\sum_{i=k_{n}\left(u_{n}+v_{n}\right)+1}^{n} \Xi_{n, i} \text {. }
\end{aligned}
$$

Then, we obtain the decomposition

$$
\begin{aligned}
Z_{n}=\sum_{i=1}^{n} \Xi_{n, i} & =\sum_{m=0}^{k_{n}-1} U_{m}+\sum_{m=0}^{k_{n}-1} V_{m}+W_{m} \\
& =S_{n, 1}+S_{n, 2}+S_{n, 3} .
\end{aligned}
$$

Now, let start the proof of theorem 2. The main idea is to show that as $n \longrightarrow \infty$,

$$
\begin{aligned}
\mathbb{E}\left[S_{n, 2}^{2}\right] & \rightarrow 0 \\
\mathbb{E}\left[S_{n, 3}^{2}\right] & \rightarrow 0 \\
\mid \mathbb{E}\left[\exp \left(i u S_{n, 1}\right)-\prod_{m=0}^{k_{n}-1} \mathbb{E}\left[\exp \left(i u U_{m}\right)\right] \mid\right. & \rightarrow 0 \\
\mathbb{E}\left[U_{m}^{2}\right] & \longrightarrow \vartheta_{\ell}\left(t_{1}, t_{2}\right) \\
\sum_{m=0}^{k_{n}-1} \mathbb{E}\left[U_{m}^{2} I\left\{\left|U_{m}\right|>\varepsilon \vartheta_{\ell}\left(t_{1}, t_{2}\right)\right\}\right] & \longrightarrow 0, \text { for every } \varepsilon>0 .
\end{aligned}
$$

Remark: Relations (32) and (33) imply that $S_{n, 2}$ and $S_{n, 3}$ are asymptotically negligible; (34) and (35) show that the summands $\left\{U_{m}\right\}$ in $S_{n, 1}$ are asymptotically independent, verifying that the sum of their variances tends to $\vartheta_{\ell}\left(t_{1}, t_{2}\right)$. Expression (36) is the Lindeberg-Feller's condition for asymptotic normality of $S_{n, 1}$ under dependence. Asymptotic normality of $Z_{n}$ is a consequence of equations (34)-(35):

$$
Z_{n} \stackrel{\mathcal{D}}{\longrightarrow} \mathcal{N}\left(0, \vartheta_{\ell}\left(t_{1}, t_{2}\right)\right)
$$

\section{- Proof of (32)}

$$
\begin{aligned}
\mathbb{E}\left[S_{n, 2}^{2}\right] & =\mathbb{V a r}\left(\sum_{m=0}^{k_{n}-1} V_{m}\right) \\
& =\sum_{m=0}^{k_{n}-1} \operatorname{Var}\left(V_{m}\right)+\sum_{\substack{m=0 \\
m \neq m^{\prime}}}^{k_{n}-1} \sum_{m^{\prime}=0}^{k_{n}-1} \operatorname{Cov}\left(V_{m}, V_{m^{\prime}}\right) \\
& =\mathfrak{J}_{1}+\mathfrak{J}_{2} .
\end{aligned}
$$

$\rightsquigarrow$ Concerning $\mathfrak{J}_{1}$,

we have

$$
\begin{aligned}
\operatorname{Var}\left(V_{m}\right) & =\operatorname{var}\left(\sum_{i=m\left(u_{n}+v_{n}\right)+u_{n}+1}^{(m+1)\left(u_{n}+v_{n}\right)} \Xi_{n, i}\right) \\
& =\sum_{i=m\left(u_{n}+v_{n}\right)+u_{n}+1}^{(m+1)\left(u_{n}+v_{n}\right)} \operatorname{Var}\left(\Xi_{n, i}\right)+\sum_{\substack{i=m\left(u_{n}+v_{n}\right)+u_{n}+1 \\
i \neq i^{\prime}}}^{(m+1)\left(u_{n}+v_{n}\right)} \sum_{i^{\prime}=m\left(u_{n}+v_{n}\right)+u_{n}+1}^{(m+1)\left(u_{n}+v_{n}\right)} \operatorname{Cov}\left(\Xi_{n, i}, \Xi_{n, i^{\prime}}\right) .
\end{aligned}
$$


Using the second-order stationarity and the fact that $\left\{\Xi_{i r s}\right\}$ and $\left\{\Xi_{i r^{\prime} s^{\prime}}\right\}$ are independent, (39) becomes

$$
\begin{aligned}
\operatorname{Var}\left(V_{m}\right) & =\sum_{i=1}^{v_{n}} \operatorname{Var}\left(\Xi_{n, i}\right)+\sum_{i=1}^{v_{n}} \sum_{i^{\prime}=1}^{v_{n}} \operatorname{Cov}\left(\Xi_{n, i}, \Xi_{n, i^{\prime}}\right) \\
& =v_{n} \operatorname{Var}\left(\Xi_{n, 1}\right)+\sum_{\substack{i=1 \\
i \neq i^{\prime}}}^{v_{n}} \sum_{i^{\prime}=1}^{v_{n}} \operatorname{Cov}\left(\Xi_{n, i}, \Xi_{n, i^{\prime}}\right) .
\end{aligned}
$$

First, we have

$$
\begin{aligned}
\operatorname{Var}\left(\Xi_{n, 1}\right) & =\operatorname{Var}\left(\sum_{1 \leq r \neq s \leq N}\left(\Xi_{1 r s}-\mathbb{E} \Xi_{1 r s}\right)\right) \\
& =\operatorname{Var}\left(\sum_{1 \leq r \neq s \leq N}\left(\Xi_{r s}-\mathbb{E} \Xi_{r s}\right)\right) \\
& =\sum_{1 \leq r \neq s \leq N} \operatorname{var}\left(\Xi_{r s}-\mathbb{E} \Xi_{r s}\right) \\
& =N(N-1) \operatorname{Var}\left(\Xi_{11}-\mathbb{E} \Xi_{11}\right) \\
& =N(N-1)\left\{\mathbb{E}\left(\Xi_{11}-\left(\mathbb{E} \Xi_{11}\right)^{2}\right\}\right. \\
& =N(N-1)\left\{\frac{\vartheta_{\ell}\left(t_{1}, t_{2}\right)}{n N(N-1)}(1+o(1))\right\} \\
& =\frac{\vartheta}{n}\left(t_{1}, t_{2}\right) \\
n & (1+o(1)) .
\end{aligned}
$$

Secondly,

$$
\begin{aligned}
\left|\operatorname{Cov}\left(\Xi_{n, i}, \Xi_{n, i^{\prime}}\right)\right| & \leq \frac{N(N-1)}{n}\left|\frac{1}{h_{n, K}^{|\lambda|+2}} \operatorname{Cov}\left(\Gamma_{i, 1,1}^{\ell}, \Gamma_{i^{\prime}, 1,1}^{\ell^{\prime}}\right)\right| \\
& \leq \frac{N(N-1)}{n}\left\{\vartheta_{\ell}^{2}\left(t_{1}, t_{2}\right)+o(1)\right\} \\
\sum_{i=1}^{v_{n}} \sum_{\substack{i^{\prime} i^{\prime} \\
i^{\prime}=1}}^{v_{n}}\left|\operatorname{Cov}\left(\Xi_{n, i}, \Xi_{n, i^{\prime}}\right)\right| & \leq \frac{v_{n}}{n}\left\{v_{n} N(N-1)\left[\vartheta_{\ell}\left(t_{1}, t_{2}\right)+o(1)\right]\right\} \\
& =\frac{v_{n}}{n}\{o(n)\} \\
& =v_{n} o(1) .
\end{aligned}
$$

Thirdly, replacing (41) and (42) in (40), it follows

$$
\begin{aligned}
\operatorname{Var}\left(V_{m}\right) & =v_{n} \frac{\vartheta_{\ell}\left(t_{1}, t_{2}\right)}{n}(1+o(1))+v_{n} o(1) \\
& =v_{n}\left\{\frac{\vartheta_{\ell}\left(t_{1}, t_{2}\right)}{n}(1+o(1))+o(1)\right\}
\end{aligned}
$$


At the end, $\mathfrak{J}_{1}$ in (38) is such that

$$
\begin{aligned}
\left|\mathfrak{J}_{1}\right| & \leq \sum_{m=0}^{k_{n}-1} v_{n}\left\{\frac{\vartheta_{\ell}\left(t_{1}, t_{2}\right)}{n}(1+o(1))+o(1)\right\} \\
& =k_{n} v_{n}\left\{\frac{\vartheta_{\ell}\left(t_{1}, t_{2}\right)}{n}(1+o(1))+o(1)\right\} \\
& =k_{n} v_{n} v_{n}\left\{\frac{\vartheta_{\ell}\left(t_{1}, t_{2}\right)}{n}(1+o(1))\right\} \\
& =k_{n} \frac{v_{n}}{n} \vartheta_{\ell}\left(t_{1}, t_{2}\right)(1+o(1)) \\
& =\left|\frac{n}{u_{n}+v_{n}}\right| \frac{v_{n}}{n} \vartheta_{\ell}\left(t_{1}, t_{2}\right)(1+o(1)) \\
& \sim \frac{n}{u_{n}} \frac{v_{n}}{n} \vartheta_{\ell}\left(t_{1}, t_{2}\right)(1+o(1)) \\
& =\frac{v_{n}}{u_{n}} \vartheta_{\ell}\left(t_{1}, t_{2}\right)(1+o(1)) \\
& \longrightarrow 0, \text { by }(26) .
\end{aligned}
$$

\section{$\rightsquigarrow$ Concerning $\mathfrak{J}_{2}$,}

$$
\begin{aligned}
& \mathfrak{J}_{2}=\sum_{\substack{m=0 \\
m \neq m^{\prime}}}^{k_{n}-1} \sum_{m^{\prime}=0}^{k_{n}-1} \operatorname{Cov}\left(V_{m} ; V_{m^{\prime}}\right) \\
& =\sum_{\substack{m=0 \\
m \neq m^{\prime}}}^{k_{n}-1} \sum_{m^{\prime}=0}^{k_{n}-1} \sum_{i=m\left(u_{n}+v_{n}\right)+u_{n}+1}^{(m+1)\left(u_{n}+v_{n}\right)} \sum_{i=i^{\prime}}^{\left(m^{\prime}+1\right)\left(u_{n}+v_{n}\right)} \operatorname{Cov}\left(\Xi_{n, i} ; \Xi_{n, i^{\prime}}\right) \\
& =\sum_{\substack{m=0 \\
m \neq m^{\prime}}}^{k_{n}-1} \sum_{m^{\prime}=0}^{k_{n}-1} \sum_{i=1}^{v_{n}} \sum_{i \neq i^{\prime}}^{v_{n}} \operatorname{Cov}\left(\Xi_{n, m}\left(u_{n}+v_{n}\right)+u_{n}+i ; \Xi_{n, m^{\prime}\left(u_{n}+v_{n}\right)+u_{n}+i^{\prime}}\right) \\
& =\sum_{\substack{m=0 \\
m \neq m^{\prime}}}^{k_{n}-1} \sum_{m^{\prime}=0}^{k_{n}-1} \sum_{i=1}^{v_{n}} \sum_{i \neq i^{\prime}}^{v_{n}} \operatorname{Cov}\left(\Xi_{n, \eta_{m}+i} ; \Xi_{n, \eta_{m^{\prime}}+i^{\prime}}\right)
\end{aligned}
$$

since $\left|\eta_{m}-\eta_{m^{\prime}}+i-i^{\prime}\right| \geq u_{n}$ then we reduce the sums and we write

$$
\begin{aligned}
& \left|\mathfrak{J}_{2}\right| \leq \sum_{i=\sum_{i-i^{\prime} \mid \geq u_{n}}}^{n} \sum_{i=1}^{n}\left|\operatorname{Cov}\left(\Xi_{n, i}, \Xi_{n, i^{\prime}}\right)\right| \\
& \leq \frac{N(N-1)}{n h_{n, K}^{|\lambda|+2}} 8 C\left[h_{n, K}^{|\lambda|+2}\right]^{2 / \delta} \sum_{\ell=1}^{\infty} \ell^{a}[\alpha(\ell)]^{1-2 / \delta} . \\
& =\frac{8 C N(N-1)}{n h_{n, k}^{(|\lambda|+2)(1-2 / \delta)}} \sum_{\ell=1}^{\infty} \ell^{a}[\alpha(\ell)]^{1-2 / \delta} \\
& =o(1) \text { by (26). }
\end{aligned}
$$

Therefore $\mathfrak{J}_{2} \longrightarrow 0$, as $n \longrightarrow+\infty$.

Combining (44) and (45), it follows that $\mathbb{E}\left[S_{n, 2}^{2}\right] \longrightarrow 0$ and

$$
S_{n, 2} \longrightarrow 0 \text { in probability. }
$$

This achieves the proof of (32).

- Proof of (33) Using the same arguments as in the proof of (32), one has 


$$
\begin{aligned}
\mathbb{E}\left[S_{n, 3}^{2}\right] & =\operatorname{Var}\left(\sum_{m=0}^{k_{n}-1} U_{m}\right) \\
& \leq \frac{u_{n}+v_{n}}{n}\left\{\vartheta_{\ell}\left(t_{1}, t_{2}\right)+o(1)\right\} \\
& \sim \frac{u_{n}}{n}\left\{\vartheta_{\ell}\left(t_{1}, t_{2}\right)+o(1)\right\} . \\
& \longrightarrow 0 .
\end{aligned}
$$

\section{- Proof of (34)}

We make use of Volkonskii \& Rozanov's (1959) Lemma (see the appendix in Masry (2005)). Note that $U_{m}$ is $\left\{\mathcal{F}_{i_{1}, \ldots, i_{u_{n}}}\right\}$-measurable with $i_{1}=m\left(u_{n}+v_{n}\right)+1$ and $i_{u_{n}}=m\left(u_{n}+v_{n}\right)+u_{n}$. Note that using that $V_{m}=\exp \left(i u U_{m}\right)$ as in the Lemma of Volkonskii \& Rozanov, we have

$$
\begin{aligned}
\left|\mathbb{E}\left\{\exp \left(i u S_{n, 1}\right)\right\}-\prod_{m=0}^{k_{n}-1} \mathbb{E}\left\{\exp \left(i u U_{m}\right)\right\}\right| & \leq 16 k_{n} \alpha\left(v_{n}+1\right) \\
& \sim 16 \frac{n}{u_{n}} \alpha\left(v_{n}+1\right) \\
& \longrightarrow 0 \text { by }(26)
\end{aligned}
$$

as $n$ goes to infinity.

\section{- Proof of (35)}

Replacing $u_{n}$ by $v_{n}$ we have

$$
\begin{aligned}
\operatorname{Var}\left(U_{m}\right) & =\operatorname{var}\left(\sum_{i=m\left(u_{n}+v_{n}\right)+1}^{m\left(u_{n}+v_{n}\right)+u_{n}} \Xi_{n, i}\right) \\
& =\sum_{i=m\left(u_{n}+v_{n}\right)+1}^{m\left(u_{n}+v_{n}\right)+u_{n}} \operatorname{Var}\left(\Xi_{n, i}\right)+\sum_{i=m\left(u_{n}+v_{n}\right)+1}^{m} \sum_{\substack{i \neq i^{\prime} \\
i^{\prime}=m\left(u_{n}+v_{n}\right)+1}}^{m} \operatorname{Cov}\left(\Xi_{n, i}, \Xi_{n, i^{\prime}}\right) \\
& =u_{n} \vartheta_{\ell}\left(t_{1}, t_{2}\right)(1+o(1)) .
\end{aligned}
$$

So

$$
\begin{aligned}
\sum_{m=0}^{k_{n}-1} \mathbb{E}\left[U_{m}^{2}\right] & =k_{n} \frac{u_{n}}{n} \vartheta_{\ell}\left(t_{1}, t_{2}\right)(1+o(1)) \\
& \sim \frac{u_{n}}{u_{n}} \vartheta_{\ell}\left(t_{1}, t_{2}\right)(1+o(1)) \\
& \longrightarrow \vartheta_{\ell}\left(t_{1}, t_{2}\right) .
\end{aligned}
$$

\section{- Proof of (36)}

We first establish the asymptotic normality (37) for the particular case where $\psi_{\ell}$ is bounded. The case of $\psi_{\ell}$ possibly unbounded is then establish by using a truncation argument. Let $\tau_{n}$ be a fixed truncation point. We can replace $\psi_{\ell}\left(T_{i r}, T_{i s}, U_{i r}, U_{i s}\right)$ with the truncated process $\psi_{\ell}\left(T_{i r}, T_{i s}, U_{i r}, U_{i s}\right) \mathbb{I}\left\{\left|\psi_{\ell}\left(T_{i r}, T_{i s}, U_{i r}, U_{i s}\right)\right| \leq \tau_{n}\right\}$ in $\left(U_{i r}, U_{i s}\right)$. Denote

$$
\begin{aligned}
\Xi_{i r s}^{\tau_{n}} & =\frac{1}{\sqrt{n N(N-1) h_{n, K}^{|\lambda|+2}}} \psi_{\ell}\left(T_{i r}, T_{i s}, U_{i r}, U_{i s}\right) \mathbb{I}\left\{\left|\psi_{\ell}\left(T_{i r}, T_{i s}, U_{i r}, U_{i s}\right)\right| \leq \tau_{n}\right\} \\
& \times K\left(\frac{t_{1}-T_{i r}}{h_{n, K}}, \frac{t_{2}-T_{i s}}{h_{n, K}}\right), \\
\Xi_{n, i}^{\tau_{n}} & =\sum_{1 \leq r \neq s \leq N}\left(\Xi_{i r s}^{\tau_{n}}-\mathbb{E} \Xi_{i r s}^{\tau_{n}}\right) .
\end{aligned}
$$


Define $Z_{n}^{\tau_{n}}=\sum_{i=1}^{n} \Xi_{n, i}^{\tau_{n}}$ and

$$
Z_{n}^{* \tau_{n}}=\sum_{i=1}^{n}\left(\Xi_{n, i}-\Xi_{n, i}^{\tau_{n}}\right)=\sum_{i=1}^{n} \Xi_{n, i} \mathbb{I}\left\{\left|\psi_{\ell}\left(T_{i r}, T_{i s}, U_{i r}, U_{i s}\right)\right|>\tau_{n}\right\} .
$$

Since $\left|\psi_{\ell}\left(T_{i r}, T_{i s}, U_{i r}, U_{i s}\right)\right| \leq \tau_{n}$ and from (H.5), it follows that

$$
\left|\Xi_{n, i}^{\tau_{n}}\right| \leq 2 C \frac{N(N-1) \tau_{n}}{\sqrt{n N(N-1) h_{n, K}^{|\lambda|+2}}}
$$

and

$$
\max _{0 \leq m \leq k_{n}-1}\left|U_{m}^{\tau_{n}}\right| \leq 2 C \frac{N(N-1) u_{n} \tau_{n}}{\sqrt{n N(N-1) h_{n, K}^{|\lambda|+2}}} .
$$

Therefore if we take $\tau_{n}$ and $u_{n}$ such that

$$
u_{n} \tau_{n}=\frac{n^{1 / 2} h_{n, K}^{|\lambda|+3}}{(N(N-1))^{1 / 2}},
$$

then,

$$
\max _{0 \leq m \leq k_{n}-1}\left|U_{m}^{\tau_{n}}\right| \leq 2 C \frac{N(N-1) u_{n} \tau_{n}}{\sqrt{n N(N-1) h_{n, K}^{|\lambda|+2}}} \longrightarrow 0 .
$$

Hence, for $n$ sufficiently large, the set $\left\{\left|U_{m}^{\tau_{n}}\right|>\epsilon \vartheta_{\ell}\left(t_{1}, t_{2}\right)\right\}$ becomes empty for all $\varepsilon>0$. Thus, $\mathbb{P}\left(\left|U_{m}^{\tau_{n}}\right|>\varepsilon \vartheta_{\ell}\left(t_{1}, t_{2}\right)\right) \longrightarrow 0$ for large $n$, for all $\varepsilon>0$. So

$$
\sum_{m=0}^{k_{n}-1} \mathbb{E}\left[U_{m}^{2} \mathbb{I}\left\{\left|U_{m}\right|>\varepsilon \vartheta_{\ell}\left(t_{1}, t_{2}\right)\right\}\right]=0, \text { for all } \varepsilon>0 .
$$

Hence

$$
Z_{n}^{\tau_{n}} \stackrel{\mathcal{D}}{\longrightarrow} \mathcal{N}\left(0, \vartheta_{\ell, \tau_{n}}\left(t_{1}, t_{2}\right)\right)
$$

In order to complete the proof, namely to establish (37) for the general case, it suffices to show that as first $n \longrightarrow+\infty$ and $\tau_{n} \longrightarrow+\infty$ (see Masry, 2005 or Fan \& Masry, 1992) we have

$$
\operatorname{Var}\left(Z_{n}^{* \tau_{n}}\right) \longrightarrow 0
$$

Indeed,

$$
\begin{gathered}
\left|\mathbb{E} \exp \left\{i u Z_{n}\right\}-\exp \left\{-u^{2} \vartheta_{\ell}\left(t_{1}, t_{2}\right) / 2\right\}\right| \\
=\mid \mathbb{E} \exp \left\{i u\left(Z_{n}^{\tau_{n}}+Z_{n}^{* \tau_{n}}\right)\right\}-\exp \left\{-u^{2} \vartheta_{\ell, \tau_{n}}\left(t_{1}, t_{2}\right) / 2\right\} \\
+\exp \left\{-u^{2} \vartheta_{\ell, \tau_{n}}\left(t_{1}, t_{2}\right) / 2\right\}-\exp \left\{-u^{2} \vartheta_{\ell, \tau_{n}}\left(t_{1}, t_{2}\right) / 2\right\} \mid \\
\leq\left|\mathbb{E} \exp \left\{i u Z_{n}^{\tau_{n}}\right\}-\exp \left\{-u^{2} \vartheta_{\ell, \tau_{n}}\left(t_{1}, t_{2}\right) / 2\right\}\right|+\mathbb{E}\left|\exp \left\{i u Z_{n}^{* \tau_{n}}\right\}-1\right| \\
+\left|\exp \left\{-u^{2} \vartheta_{\ell, \tau_{n}}\left(t_{1}, t_{2}\right) / 2\right\}-\exp \left\{-u^{2} \vartheta_{\ell}\left(t_{1}, t_{2}\right) / 2\right\}\right| .
\end{gathered}
$$

Letting $n \longrightarrow+\infty$, the first term goes to zero by (50), for every $\tau_{n}>0$; the second term converges to zero by (51), because first $n \longrightarrow+\infty$ and then $\tau_{n} \longrightarrow+\infty$; the third term goes to zero as $\tau_{n} \longrightarrow+\infty$ by the dominated convergence theorem.

Therefore, it remains to prove (51). Note that by (50), $Z_{n}^{* \tau_{n}}$ has the same structure as $Z_{n}^{\tau_{n}}$ except that $\Xi_{n, i}^{\tau_{n}}$ is replaced by $\left(\Xi_{n, i}-\Xi_{n, i}^{\tau_{n}}\right)$. Applying the Lemma 2.3 in Fan \& Masry (1992) or using the same arguments as in Masry (2005) we conclude that, for all fixed $\tau_{n}>0$, one has (51).

Then, it suffices to choose $\tau_{n}$ sufficiently large, such that the non-truncated part becomes asymptotically negligible. 
The following theorem provides the first main result of the paper.

Theorem 3 Under assumptions of theorems 1 and 2, we have

$$
\sqrt{n N(N-1) h_{n, K}^{|\lambda|+2}}\left[\hat{\Theta}_{n, \ell}\left(t_{1}, t_{2}\right)-\theta_{\ell}\left(t_{1}, t_{2}\right)\right] \stackrel{\mathcal{D}}{\longrightarrow} \mathcal{N}\left(\mathbf{B}\left(t_{1}, t_{2}\right), \vartheta_{\ell}\left(t_{1}, t_{2}\right)\right)
$$

Proof. According to the Proposition 2, p 243 (Müller \& Song, 1993), Theorem 3 is a consequence of theorem 1 and theorem 2. $\square$

Now, we extend the general result (Theorem 2 of Yao, 2007) under alpha-mixing conditions. Then, additional assumptions on the bandwidth are given in assumptions (H.2).

Let $H: \mathbb{R}^{L} \longrightarrow \mathbb{R}$ be a function with continuous second order derivatives. If we denote the gradient vector $\left(\frac{\partial H}{\partial x_{1}}(v), \ldots, \frac{\partial H}{\partial x_{L}}(v)\right)^{T}$ by $D H(v)$, and let

$$
\begin{aligned}
\tilde{\mathbf{B}}\left(t_{1}, t_{2}\right) & =\frac{(-1)^{|k|} a}{k !} \sum_{\ell=1}^{L}\left\{\int_{\mathbb{R}^{2}} v^{k_{1}} w^{k_{2}} K(v, w) d v d w \frac{d^{|k|}}{d t_{1}^{k_{1}} d t_{2}^{k_{2}}} \int_{\mathbb{R}^{2}} \psi_{\ell}\left(t_{1}, t_{2}, u_{1}, u_{2}\right) g_{1}\left(t_{1}, t_{2}, u_{1}, u_{2}\right) d u_{1} d u_{2}\right\} \\
& \times\left\{\frac{\partial H}{\partial \theta_{L}}\left(\theta_{1}, \ldots, \theta_{L}\right)^{T}\right\}
\end{aligned}
$$

and

$$
\begin{aligned}
\vartheta_{\ell, \ell^{\prime}}\left(t_{1}, t_{2}\right) & =\|K\|_{2}^{2} \int_{\mathbb{R}^{4}} \psi_{\ell}\left(t_{1}, t_{2}, u_{1}, u_{2}\right) \psi_{\ell^{\prime}}\left(t_{1}, t_{2}, u_{1}, u_{2}\right) g_{2}\left(t_{1}, t_{2}, t_{1}, t_{2}, u_{1}, u_{2}, u_{1}^{\prime}, u_{2}^{\prime}\right), d u_{1} d u_{2} d u_{1}^{\prime} d u_{2}^{\prime}, \\
V & =\left(\vartheta_{\ell, \ell^{\prime}}\left(t_{1}, t_{2}\right)\right)_{1 \leq \ell, \ell^{\prime} \leq L} \text { the variance-covariance matrix, }
\end{aligned}
$$

then the second main result of the paper follows.

Theorem 4 Assume that assumptions of theorem 3 hold. Then

$$
\sqrt{n N(N-1) h_{n, K}^{|\lambda|+2}}\left[H\left(\hat{\Theta}_{1 n}, \ldots, \hat{\Theta}_{L n}\right)-H\left(\theta_{1}, \ldots, \theta_{L}\right)\right] \stackrel{\mathcal{D}}{\longrightarrow} \mathcal{N}\left(\tilde{\mathbf{B}}\left(t_{1}, t_{2}\right),\left[D H\left(\theta_{1}, \ldots, \theta_{L}\right)\right]^{T} V\left[D H\left(\theta_{1}, \ldots, \theta_{L}\right)\right]\right)
$$

Proof. A $L$-dimensional Taylor expansion of $H$ around $\left(m_{1}, \ldots, m_{L}\right)^{T}$ of order 1 combined with (8) gives

$$
\sqrt{n N(N-1) h_{n, K}^{|\lambda|+2}}\left[H\left(\mathbb{E} \hat{\Theta}_{1 n}, \ldots, \hat{\Theta}_{L n}\right)-H\left(\theta_{1}, \ldots, \theta_{L}\right)\right] \stackrel{\mathbb{P}}{\longrightarrow} \tilde{\mathbf{B}}\left(t_{1}, t_{2}\right) .
$$

Applying the Cramér-Wold device to (24) it comes

$$
\sqrt{n N(N-1) h_{n, K}^{|\lambda|+2}}\left(H\left(\hat{\Theta}_{1 n}, \ldots, \hat{\Theta}_{L n}\right)-H\left(\mathbb{E} \hat{\Theta}_{1 n}, \ldots, \mathbb{E} \hat{\Theta}_{L n}\right)\right) \quad \longrightarrow \quad \mathcal{N}\left(0,\left[D H\left(\theta_{1}, \ldots, \theta_{L}\right)\right]^{T} V\left[D H\left(\theta_{1}, \ldots, \theta_{L}\right)\right]\right)
$$

Finally, (54) and (55) lead to (53).

\section{Acknowledgements}

We are grateful to the anonymous referees whose suggestions helped us to improve the quality of the paper.

\section{References}

Fan, J., \& Masry, E. (1992). Multivariate regression estimation with errors-in-variable : Asymptotic normality for mixing processes. Journal of Multivariate Analysis, 43, 237-271. https://doi:10.1016/0047-259X(92)90036-F.

Hal1, P., Müller, H. G., \& Yao, F. (2008). Modeling Sparse Generalized Longitudinal Observations With Latent Gaussian Processes, J. R. Statist. Soc. B 70 Part 4 pp. 703-723.

Hall, P., \& Heyde, C. C. (1980). Martingale Limit Theory and Its Applications. Academic Press, New York,

Masry, E. (2005). Nonparametric regression estimation for dependent functional data: asymptotic normality . Stochastic Processes and their Applications, 115, 155-177. https://dx.doi.org/10.1016/j.spa.2004.07.006. 
Müller, H. G., \& Song, K. S. (1993). Identity reproducing multivariate nonparametric regression. Journal of Multivariate Analysis, 46, 237-253.

Müller, H. G. (1984). Smooth optimum kernel estimators of densities, regression curves and modes. Annals of Statistics, $12,766-774$.

Nadaraya, E. A. (1964). On estimating regression. Theory of Probability and Its Applications, 9, 141-142.

Rosenblatt, M. (1956). A central limit theorem and a strong mixing condition. Proc. Nat. Acad. Sci. U. S. A., 42, 43-47.

Soro, B., \& Hili, O. (2012). Nonparametric estimation of a multiple order conditional within-subject covariance function for a continuous times univariate stochastic process. C. R. Acad. Sci. Paris, Ser I ,350, 1055-1058. https://dx.doi.org/10.1016/j.crma.2012.10.026

Stone, C. J. (1977). Consistent nonparametric regression. Annals of Statistics, 5, 595-645.

Volkonskii, V. A., \& Rozanov, Y. A. (1959). Some limit theorems for random functions. Theory Probab. Appl., 4, 178-197.

Watson, G. S. (1964). Smooth regression analysis. Sankya, Series A26, 359-372.

Yao, F. (2007). Asymptotic distributions of nonparametric regression estimators for longitudinal or functional data. Journal of Multivariate Analysis, 98, 40-56. https://doi:10.1016/j.jmva.2006.08.007

\section{Copyrights}

Copyright for this article is retained by the author(s), with first publication rights granted to the journal.

This is an open-access article distributed under the terms and conditions of the Creative Commons Attribution license (http://creativecommons.org/licenses/by/4.0/). 